\title{
Synergistic effects of mifepristone on the cytotoxicity of cisplatin in cervical carcinoma cell lines and tumors grown in athymic mice Rafael Jurado*, Angélica López-Flores, Aarón Álvarez-Figueroa and Patricia García-López
}

\author{
Address: Subdirección de Investigación Básica, Instituto Nacional de Cancerología \\ Email: Rafael Jurado* - fcojl@yahoo.com \\ * Corresponding author
}

from 24th Annual Meeting of the National Cancer Institute of Mexico Mexico City, Mexico. 14-17 February 2007

Published: 5 February 2007

BMC Cancer 2007, 7(SuppI I):AI8 doi:I0.I I86/I47I-2407-7-SI-AI8

This article is available from: http://www.biomedcentral.com/I47I-2407/7/SI/AI8

(c) 2007 Jurado et al; licensee BioMed Central Ltd.

\section{Background}

Cisplatin and its derivatives are important drugs in cancer therapy. It has been widely used for its potent cytotoxic effects upon a variety of tumors types including cervical carcinoma. However, the administration of cisplatin is associated with serious side effects, including nephrotoxic and neurotoxic events. There have been several studies aimed at finding drugs able to potentiate the antiproliferative effects of cisplatin without increasing the already serious side effects, but the search has not been successful.

In this work we investigated the ability of a progesterone antagonist, mifepristone, to modulate the cytotoxic effects of cisplatin in cancer cell lines and in a model of cervix cancer in athymic mice.

\section{Materials and methods}

The effect of cisplatin alone and cisplatin combined with mifepristone on cellular death was studied using an assay based on a tetrazolium dye, XTT. Before and after treatment with mifepristone, the intracellular accumulation of cisplatin in cancer cells and tumors of mice receiving treatment was evaluated by HPLC, the expression of Bcl-2 gene was assessed by RT-PCR and western blotting. Tumors measurements were performed weekly using vernier calipers.

\section{Results}

Analysis of the data by the isobologram method shows that the combination of mifepristone and cisplatin pro- duced a synergistic effect in cervical cancer cells. The effect of mifepristone on the cytotoxicity of cisplatin could be mediated, at least partially, by increase of intracellular cisplatin accumulation and the down-regulation of the $\mathrm{Bcl}-2$ expression. The in vivo studies showed that the combination of these agents had a significant antitumor activity against xenograft tumors.

\section{Conclusion}

Our results suggest that mifepristone can improve the efficacy of cisplatin in cancer cells and this anti-hormonal drug therapy may be a candidate for further evaluation in combination with antineoplastic drugs in the treatment of cancer.

\section{Acknowledgements}

This work was support by the CONACYT (México) Grant No. P44I76-M 